\title{
TLR3 in antiviral immunity: key player or bystander?
}

\author{
Martina Schröder and Andrew G. Bowie
}

Viral Immune Evasion Group, Department of Biochemistry, Trinity College Dublin, College Green, Dublin 2, Ireland

Toll-like receptor 3 (TLR3), which recognizes doublestranded (ds)RNA, was the first identified antiviral TLR and, because dsRNA is a universal viral molecular pattern, TLR3 has been assumed to have a central role in the host response to viruses. However, this role has recently been questioned by in vivo studies and the discovery of several other antiviral pattern-recognition receptors. In this review, the function of TLR3 in the context of these other receptors, namely TLR7, 8 and 9 and the newly identified dsRNA-receptor retinoic-acid inducible gene-I (RIG-I) is discussed. Also, recent research concerning the expression profile of TLR3, its evasion by viruses and a potential role in crosspriming is addressed, which reveals a clearer appreciation of the contribution of TLR3 to antiviral immunity.

\section{Introduction}

Toll-like receptors (TLRs) recognize different pathogenassociated molecular patterns (PAMPs), leading to the activation of an innate immune response and the shaping of the subsequent adaptive immune response. The first described ligands for TLRs were of bacterial origin, such as lipopolysaccharide (for TLR4) or peptidoglycan (for TLR2), but since the discovery of double-stranded (ds) RNA as the ligand for TLR3 it has been recognized that TLRs also have a role in the host defense against viruses [1]. It is now clear that TLR3 can mediate responses to the synthetic analog of viral dsRNA, polyriboinosinic: polyribocytidylic acid [poly(I:C)], which has been used extensively in experimental studies to mimic viral infection. Apart from constituting the genome of one class of viruses, dsRNA is also generated during the life cycle of most other viruses. Therefore, it has been assumed that TLR3 would have a key role in antiviral immunity. However, recent studies have questioned this [2], and it is now clear that other TLRs, such as TLR7, 8 and 9, have fundamental roles in the response to distinct viruses [3-8]. Furthermore, an additional pattern-recognition receptor (PRR) for dsRNA, retinoic-acid inducible gene-I (RIG-I), has recently been identified [9] and seems to be emerging as a key player in the induction of an interferon response by viruses. In this review, therefore, the current data on TLR3 is summarized and an attempt to contextualize its role in antiviral immunity is made.

Corresponding author: Bowie, A.G. (agbowie@tcd.ie).

Available online 18 July 2005
TLR3 signaling pathway

Similar to TLR4 [10], engagement of the TLR3 signaling pathway leads to the activation of two transcription factors with central roles in innate immunity: NF- $\mathrm{B}$ and interferon (IFN)-regulatory factor 3 (IRF3) [11]. IRF activation by TLRs is reviewed in detail by Moynagh, also in this issue [12]. However, there are a few major differences compared to the other TLR signaling pathways. For example, in contrast to all other known TLRs, TLR3 does not recruit the adaptor molecule MyD88 but depends solely on the alternative adaptor Toll-interleukin-1 (IL-1) receptor-resistance (TIR) domain-containing adaptor inducing IFN- $\beta$ (TRIF) [13-16]. Furthermore, IL-1 receptor-associated kinase 1 (IRAK1) and IRAK4 are also dispensable for TLR3 signaling [17]. Recently, it has been demonstrated that TLR3 is phosphorylated at two tyrosine residues on activation, and that phosphatidylinositol 3-kinase (PI3K; PtdIns 3-kinase) is recruited subsequently to these residues [18], a process that seems to be required for full activation of IRF3 $[19,20]$. The only other TLR that has been shown to be phosphorylated and to recruit PI3K is TLR2 [21]; it therefore remains an open question whether this mechanism also applies to the other TLRs. The other essential step for IRF3 activation is mediated by the recruitment of TANK [tumor necrosis factor (TNF) receptor-associated factor (TRAF) family member-associated NF- $\mathrm{NB}$ activator]-binding kinase-1 (TBK-1) to TRIF [11,22].

$\mathrm{NF}-\kappa \mathrm{B}$ activation seems to be at least partially mediated by receptor-interacting protein-1 (RIP1) recruitment [23]. TRAF6 has also been implicated in NF- $\mathrm{KB}$ activation by TRIF [11,13,24]; however, its role has now been questioned by a study using macrophages from TRAF $6^{-1-}$ mice, in which activation of NF- $\kappa \mathrm{B}$ by $\operatorname{poly}(\mathrm{I}: \mathrm{C})$ was normal in the knockout cells, as was induction of two $\mathrm{NF}-\kappa \mathrm{B}-$ dependent genes [25]. Their results are in contrast to the earlier results by Jiang et al., who found that TRAF6 was required for NF- $\mathrm{KB}$ activation in murine embryonic fibroblasts [13]. Therefore, the involvement of TRAF6 in TRIF-induced NF- $\kappa \mathrm{B}$ activation is controversial, and it is possible that the requirement for TRAF6 is cell-type specific or that TRAF6 and RIP1 mediate NF- $\mathrm{kB}$ activation at different stages of the response. The interaction with RIP1 also links TRIF to the apoptotic cascade [26]. The pro-apoptotic effect is further mediated by Fas-associated death domain (FADD) and caspase- 8 and it is conceivable that RIP1 engages FADD through its death domain $[24,26]$. The current knowledge of the 
signaling pathways initiated by TLR3 is summarized in Figure 1.

TRIF is not a TLR3-specific adaptor molecule but is also involved in IRF3 activation by TLR4. However, its recruitment to TLR4 is mediated by an additional adaptor molecule, TRIF-related adaptor molecule (TRAM) [27]. Therefore, the TRIF pathway is differentially activated by TLR3 and TLR4. Consistent with this, IFN-stimulated response element induction by TLR4, but not by TLR3, requires the NF-кB subunit p65 [28]. In addition, TLR3 engagement leads to a much stronger induction of type I IFNs than does TLR4 signaling [29]. However, TBK1 mediates both TLR4- and TLR3-induced IRF3 activation [30], so the observed differences between TLR3- and TLR4-induced IRF3 activation might be quantitative rather than qualitative [31].

\section{TLR3 in viral infection}

The first study showing that TLR3 signaling was triggered by dsRNA used not only poly(I:C) but also purified genomic RNA from reovirus to stimulate the receptor [1]. Therefore, there is little doubt that TLR3 responds to viral as well as to synthetic dsRNA. But what role does it have in the response to viral infection? Because dsRNA is a universal viral PAMP, it had been assumed that TLR3 would have a key role in antiviral immunity. Indeed, TLR3 has been implicated in the response to several viruses $[1,14,32-34]$, as discussed later.

Wang et al. demonstrated that, in infections with the single-stranded (ss)RNA virus West Nile virus, a peripheral inflammatory response is initiated through TLR3, leading to disruption of the blood-brain barrier, which enables virus entry into the brain [34]. Therefore, $T L R 3^{-1-}$ mice were more resistant to lethal West Nile virus infection, so that in this case the virus appeared to benefit from its interaction with TLR3 [34]. TLR3 also seems to be the TLR most strongly expressed in the brain, specifically in astrocytes and glioblastoma cell lines $[35,36]$, and there has been a description of a TLR3 isoform that seems to be exclusively expressed in the brain [36]. These findings might indicate that TLR3 has a specific role in the brain and/or in the response to encephalitogenic viruses but this should be further investigated.

Apart from this somewhat specific case, it was demonstrated that cytokines are induced in a TLR3-dependent manner in bronchial epithelial cell lines, on infection with the ssRNA viruses respiratory syncytial virus or influenza, although no animal models were used to verify a role for TLR3 in vivo [32,33]. Interestingly, however, Rudd et al. found that respiratory syncytial virus-induced CXCL10 and CCL5 production, but not CXCL8 production or viral replication, were impaired in the absence of TLR3 [32].

It was shown that Lps2-mice, which have a loss-offunction mutation in the Trif gene, show a higher rate of mortality in murine cytomegalovirus (MCMV) infections [14], and in a recent study, a role for TLR3 in the response to MCMV was confirmed using TLR3 ${ }^{-1-}$ mice [6]. However, a study by Edelmann et al. questioned a universal role for TLR3 in the antiviral response, claiming

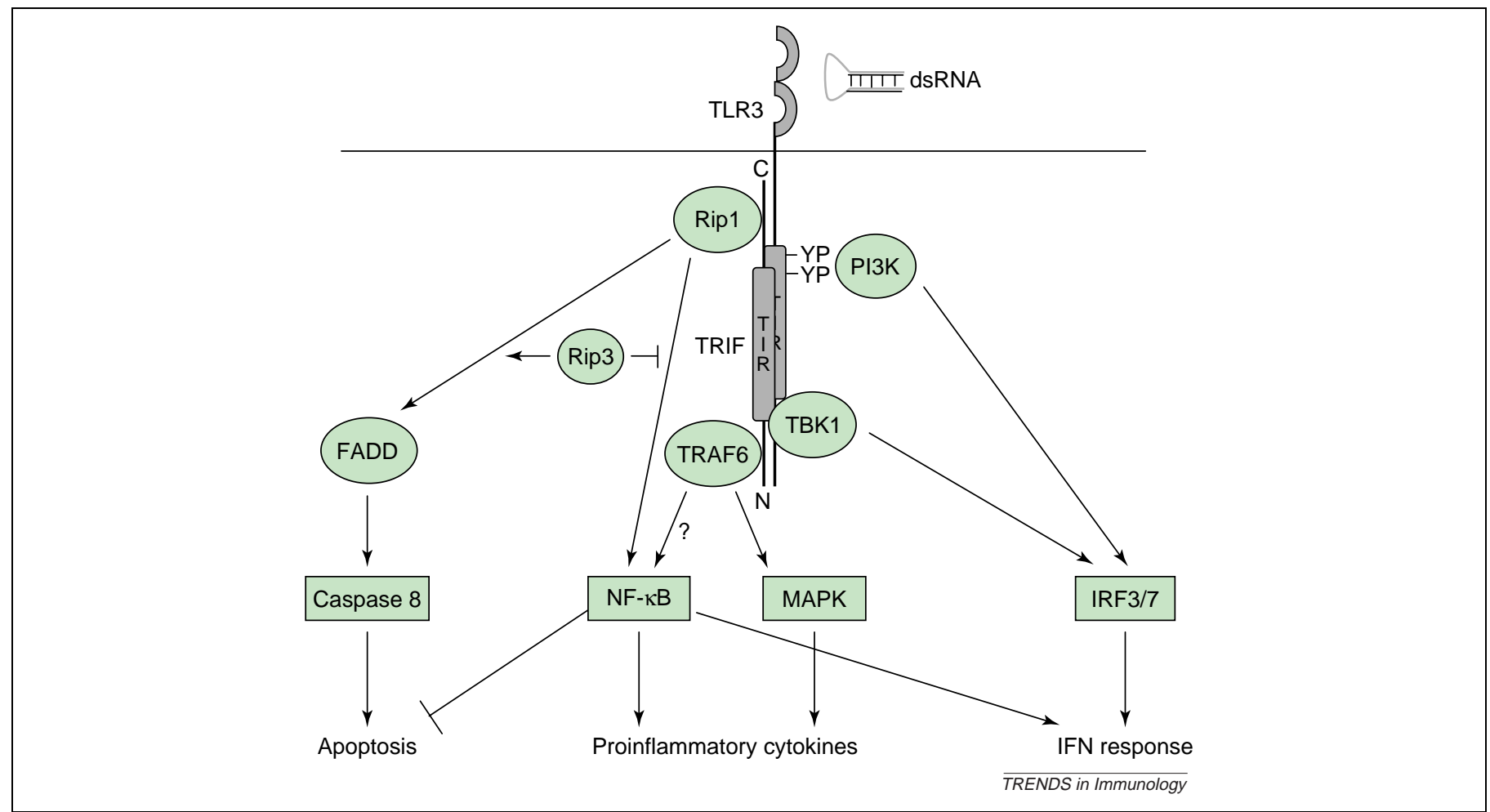

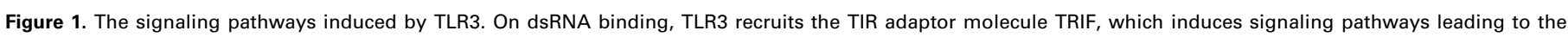

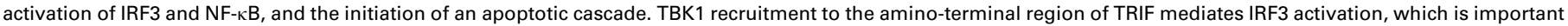

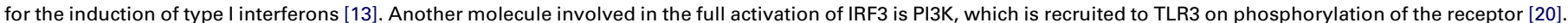

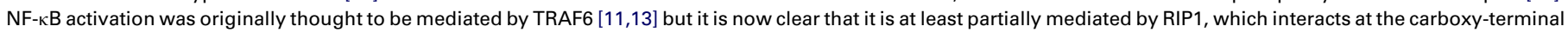

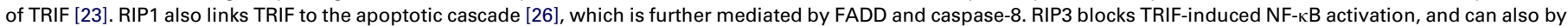

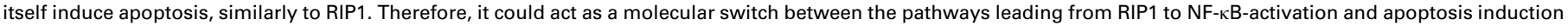

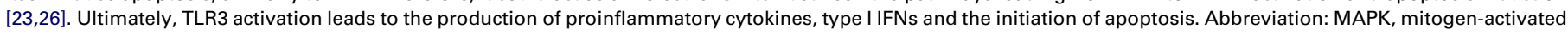
protein kinase. 
that it is dispensable for both viral pathogenesis and the generation of a host adaptive immune response in MCMV, vesicular stomatitis virus (VSV), lymphocytic choriomeningitis virus and reovirus infection [2]. Therefore, the role of TLR3 in the response to viral infections, especially to MCMV infection, is controversial, and later a potential role for TLR3 will be defined by comparing it with other TLR-dependent and -independent antiviral responses.

\section{Alternative antiviral PRRs}

With the discovery that unmethylated viral CpG-DNA (from herpes simplex virus-2) can stimulate TLR9 [4] and the identification of ssRNA (from influenza virus and HIV-1) as the ligand for TLR7 [37,38], it seems that a whole class of PRRs for viral nucleic acids is emerging. TLR7 is involved in the response to the ssRNA viruses VSV and influenza [5], whereas TLR9 has a role in responding to the dsDNA viruses MCMV [6], herpes simplex virus-1 (HSV-1) and HSV-2 [3,4,8].

Before the discovery of TLR3, it was well known that cells detected viral dsRNA through dsRNA-dependent protein kinase (PKR), which, in response, inhibits host translation by phosphorylating the initiation factor, the $\alpha$ subunit of eukaryotic protein synthesis initiation factor 2 , and, similar to TLR3, activates NF-кB [39]. Indeed, the fact that $P K R^{-1-}$ cells still signaled in response to poly(I:C) led to the identification of TLR3, which was thought to provide the missing link [1]. However, it soon became clear that cells must have yet additional mechanisms for the response to dsRNA with the finding that $T L R 3^{-1-}$ or $T R I F^{-1-}$ cells still showed a response to poly(I:C) which could not be completely attributed to PKR [40,41].

Yoneyama et al. identified the IFN-inducible DExD/H box RNA helicase RIG-I as a novel intracellular PRR for dsRNA [9]. Apart from its helicase domain, RIG-I also contains a tandem caspase recruitment domain (CARD), which transmits signals leading to $\mathrm{NF}-\mathrm{\kappa B}$ and IRF3 activation [9]. The authors demonstrated that RIG-I is essential for IFN induction by Newcastle disease virus and this also seems to be the case for Sendai and hepatitis $\mathrm{C}$ virus (HCV) infections [42,43]. Furthermore, replication of VSV and encephalomyocarditis virus was inhibited in RIG-I overexpressing cells [9]. The RIG-I pathway seems to be independent of the TLR3 pathway because a dominant negative form of RIG-I did not block TLR3- or TRIF-induced IRF3 activation. Similarly, TRIF and TLR3 were found to be dispensable for RIG-I signaling [9]. It is likely that RIG-I interacts with other CARD domaincontaining proteins to relay its signaling; however, these remain to be identified. Along with RIG-I, a homologous CARD domain-containing helicase, melanoma differentiation-associated gene-5 (mda-5), was identified and shown to have similar effects in dsRNA signaling [9]. Therefore, the response to dsRNA is multifaceted and is likely to become even more complex. Notably, a mouse locus that had been identified to confer TRIF-independent responsiveness to poly(I:C) [41] does not correspond to either RIG-I or mda-5 [9]. Therefore, this gene product might either function in the RIG-I pathway, or represent yet another independent mechanism for the response to dsRNA.

Given the reports described earlier, it has become more difficult to define a specific role for TLR3 in the antiviral response. It is probable that viral infections, similar to bacterial infections, produce a multitude of ligands that act on different TLRs or non-TLR PRRs. Therefore, it could be simply the redundancy in the viral detection system that makes it difficult to pinpoint a role for one specific component. However, there are now clear roles for TLR7, TLR9 and RIG-I in antiviral responses. So, is TLR3 just not as important? Arguing against this assumption would be the fact that we and others have recently identified viral evasion mechanisms targeting the TLR3 pathway, and it is generally assumed that viruses, in a process of highly efficient coevolution with the host, target the key mechanisms of the antiviral response.

\section{Viral evasion of the TLR3 pathway}

We have shown recently that vaccinia virus (VV), a dsDNA virus, expresses two proteins, A46R and A52R, which target different aspects of the TLR3 signaling pathway and thereby inhibit TLR3-induced gene induction efficiently. Whereas A46R, a TIR domain-containing protein, binds to TRIF directly and inhibits poly(I:C)-induced IRF3 activation, A52R potently blocks TLR3 signaling, leading to $\mathrm{NF}-\kappa \mathrm{B}$ activation $[44,45]$. Interesting in this context is that VV replicates to a higher titer in macrophages derived from Lps2 mice [14], which implicates TRIF in the antiviral IFN response to VV, leading to the inhibition of viral replication.

Also, NS3-4a, a protease from HCV, besides targeting some as yet unknown component of the RIG-I pathway $[43,46]$, has been described recently to cleave TRIF specifically, thereby abrogating TLR3 signaling $[47,48]$. Therefore, it seems important for the virus to disrupt not only the RIG-I but also the TLR3 pathway. However, it should be noted that TRIF is also necessary for IFN induction by TLR4. Thus, certain viruses could benefit from targeting TRIF through preventing IFN induction by opportunistic bacterial infections, which would engage TLR4.

\section{Role of TLR3 in viral detection: does location hold the key?}

It is likely that the intracellular localization and cellular expression pattern of TLR3, as compared with that of RIG-I and TLR7, 8 and 9, will provide a key to answering the question about its specific role in the antiviral response. TLR3 localizes to an intracellular vesicular compartment in dendritic cells (DCs) and cannot be detected on the cell surface [49]. This compartment has been assumed to be endosomal because inhibition of endosomal acidification abrogates poly(I:C) signaling. However, Matsumoto et al. failed to see co-localization of TLR3 with endosomal or, indeed, any other organelle markers tested [49]; the exact nature of the TLR3-containing vesicles therefore needs to be clarified. In any case, TLR3 has to encounter dsRNA in these vesicles, which is probably achieved through phagocytosis of dsRNA released into the extracellular space by necrotic or virally 
lysed cells. The other possible scenario would be the exposure of a dsRNA genome during viral entry through receptor-mediated endocytosis. In contrast to TLR3, PKR, RIG-I and mda-5 are cytoplasmic and therefore much more suited for the direct recognition of dsRNA produced during viral replication. For the establishment of an adaptive immune response against virus-infected cells, it is crucial to induce DC maturation. Therefore, the relative contribution of RIG-I versus TLR3 for the response to a particular virus might, among other factors, depend on the ability of the virus to infect DCs. It is conceivable that the host response to a virus, such as Sendai virus, which infects DCs and enters the cell by plasma membrane fusion (thereby avoiding the endosomes), would rather depend on RIG-I. In fact, the host response to Sendai virus is completely independent of TLR3, 7, 8 or 9 [50] and is mediated by RIG-I [43].

It is probable, however, that dsRNA, during the course of a viral infection, comes into contact with both the cytoplasmic receptors and TLR3. So, why does a cell need different mechanisms to recognize dsRNA? This is particularly perplexing because the signaling pathways elicited by TLR3, PKR and RIG-I overlap significantly, in that all three activate NF- $\mathrm{B}$, whereas RIG-I and TLR3 (and possibly also PKR) activate IRF3 and IRF7 [9,13,39]. RIG-I and TLR3 even seem to depend on the same kinases, IKK- $\varepsilon$ and TBK1, to phosphorylate IRF3 [51,52]. Furthermore, apoptosis, is induced not only by TLR3 but also by PKR and probably by RIG-I [26,53-56]. Interestingly, however, different gene profiles are induced by poly(I:C) stimulation (through TLR3) or Sendai virus infection (through RIG-I), with only a couple of genes similarly induced by both pathways [57]. Therefore, it is probable that subtle differences exist in the signaling pathways of TLR3, PKR and RIG-I, which will, on further investigation, provide more insights into the specific roles of these dsRNA receptors.

\section{Different TLRs for different DC populations}

Whereas TLR3 displays a distinct intracellular localization compared with RIG-I and PKR, it is striking that TLR3, 7, 8 and 9 show a similar intracellular localization, and it therefore appears that they are all designed to detect viral nucleic acids in endosomal compartments. However, they are expressed by different DC populations: TLR3 is not expressed in plasmacytoid DCs (pDCs), which express high levels of TLR7 and TLR9. By contrast, TLR3 is expressed in myeloid DCs, specifically in human monocyte-derived DCs and CD $11^{+}$blood DCs, as well as in murine $\mathrm{CD} 8 \alpha^{+}$DCs. Importantly, this differential expression profile is reflected in distinct gene induction patterns: pDCs produce high amounts of type I IFNs (predominantly IFN- $\alpha$ ) in response to TLR7 or TLR9 engagement, whereas myeloid DCs mainly produce IL-12 and IFN- $\beta$ on TLR3 stimulation [58-60] (Figure 2). It is therefore possible to imagine that different DC subtypes are involved in different stages of the antiviral response. For example, TLR7 and TLR9 might trigger a quick IFN response, whereas TLR3 might be more important for a prolonged response and the initiation of the adaptive immune response. In this context, it is interesting to note that, in MCMV infection, IL-12 production seemed to be more dependent on TLR3, compared with type I IFN production [6]. Concerning the expression of TLRs, there appears to be some divergence between the murine and the human system; for example, murine, but not human, myeloid DCs express TLR9. This could mean that there is less redundancy in the human viral detection system, and it certainly means that data obtained in murine knockout models must be interpreted carefully with respect to the human system [58-61].

\section{TLR3 on natural killer (NK) cells}

NK cells are major players in the antiviral immune response and express TLR3 and are activated directly in response to poly(I:C) [62-64]. This could represent another important function for TLR3, especially in virus infections that heavily rely on NK cells for clearance (Figure 2). This is the case for MCMV infections, and, indeed, TLR3 contributes to the response to this virus [6]. However, NK cells also seem to be able to respond directly to other viral TLR stimuli, such as CpG-DNA [64]. Therefore, activation of NK cells might not be a unique feature of TLR3 but might similarly apply to TLR9 and possibly TLR7 and 8.

\section{Crosspriming: a more specific role for TLR3}

In the case of viruses that have no tropism for DCs, the immune system relies on crosspresentation and crosspriming for the initiation of a $\mathrm{CD}^{+}$T-cell response against the infected tissue cells. Viral antigens from these cells have to be crosspresented by antigen-presenting cells (APCs) and virally infected cells have to provide a DC maturation signal (crosspriming). Schulz et al. suggested recently that TLR3 has an important role in crosspriming [65]. They showed that poly(I:C)-transfected tissue cells stimulated the upregulation of co-stimulatory molecules on DCs more efficiently than free poly(I:C); this was attributed to a different uptake mechanism for the poly(I:C)-containing cells. Moreover, Semliki forest virusor encephalomyocarditis virus-infected cells induced DC activation efficiently in this system, showing that biological levels of dsRNA intermediates are sufficient for this response. By using TLR3 $3^{-1-}$ mice, the authors demonstrated that this crosspriming mechanism is mediated largely by TLR3. It had been suggested previously that TLR3 and TLR9, but not other TLRs, can mediate crosspresentation and -priming $[66,67]$.

In this context, it should be mentioned that Sato and Iwasaki have shown that TLR signaling through MyD88 is required for the optimal induction of a Th1 response against HSV-2 in both the infected tissue cells and the non-infected DCs [68]. A similar two-step model could apply for the recognition of dsRNA by TLR3. In fact, Lebre et al. demonstrated that the supernatant from poly(I:C)stimulated keratinocytes can prime DCs to induce a Th1 response [69]. Furthermore, type I IFNs, released from virally infected tissue cells, can also promote the crosspriming of DCs [70] (Figure 2), a mechanism that was prevented in the experimental model of Schulz et al. [65]. Therefore, it is possible that TLR3 has a more prominent role in the response to viruses that interfere successfully 


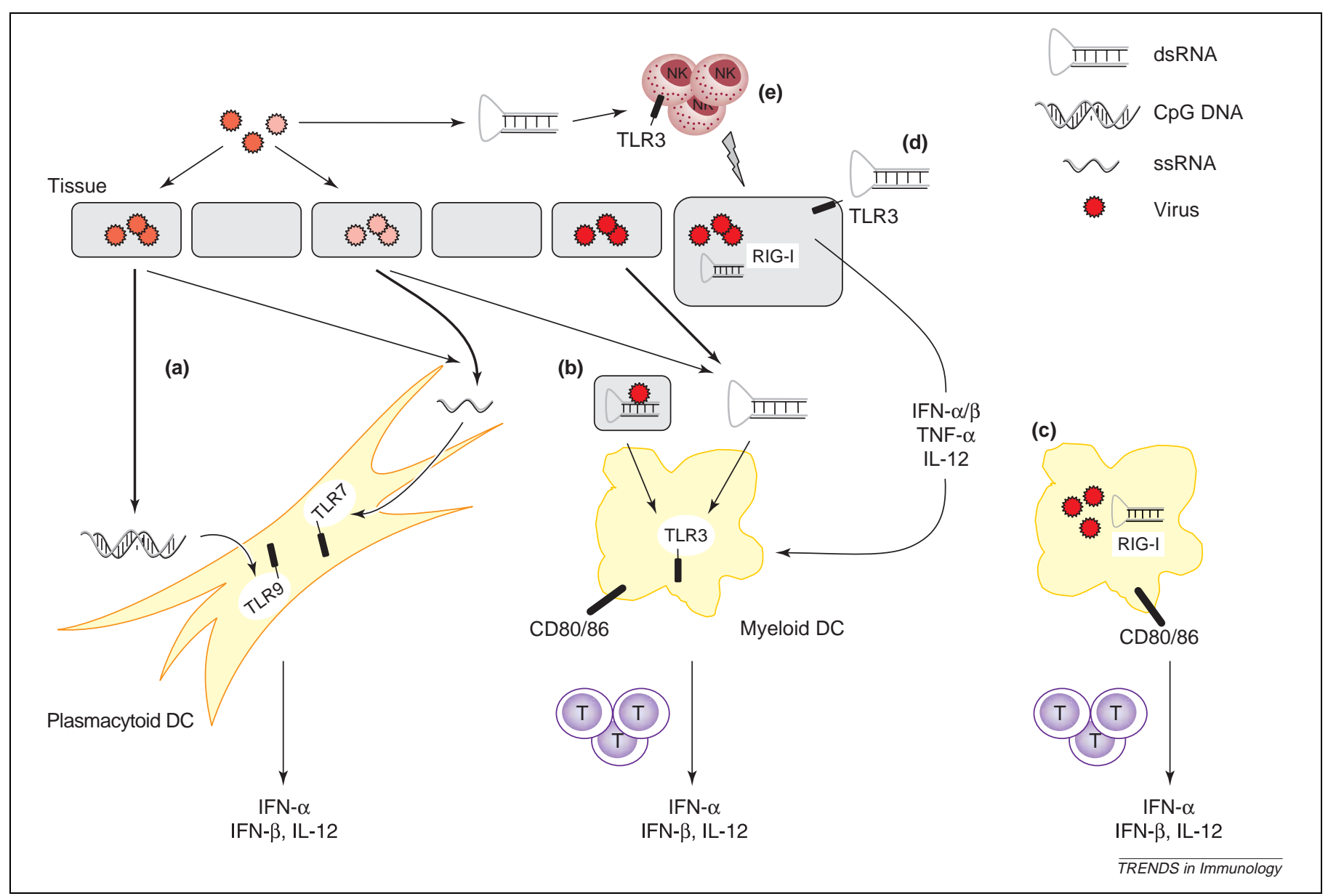

Figure 2. The immune response to viral nucleic acids. Viral nucleic acids are recognized by distinct PRRs expressed in different cell types. DCs take center stage in this response, as they are crucial for initiating an adaptive immune response. (a) Plasmacytoid DCs express TLR7 and TLR9, which recognize viral ssRNA and CpG-DNA, respectively, in an endosomal compartment. The viral nucleic acids are likely to be released from virally infected tissue cells and to be taken up through phagocytosis by the DCs. The engagement of TLR7 or TLR9 on pDCs leads to the production of type I IFNs, particularly IFN- $\alpha$. (b) By contrast, TLR3 is expressed by myeloid DCs, which produce mainly IFN- $\beta$ and IL-12 on stimulation by dsRNA $[59,60]$. The dsRNA can also either originate from lysed infected cells or it can be taken up in a cell-associated form, which induces a stronger DC maturation signal (upregulation of co-stimulatory molecules). The latter event leads to crosspriming, which is necessary to induce a CD8 ${ }^{+} \mathrm{T}_{\text {-cell }}$ response to infected tissue cells [65]. (c) In contrast to TLR3, RIG-I is expressed in the cytoplasm of cells and is therefore more suited for the recognition of dsRNA produced during viral replication [9]. Therefore, a virally infected DC can directly induce DC maturation through RIG-I, circumventing a role for TLR3. (d) DsRNA can also be recognized by tissue cells through either TLR3 or RIG-I, leading to the production of cytokines and IFNs, which can activate DCs, promote crosspriming and shape the adaptive immune response $[69,70]$. (e) Furthermore, NK cells have been shown to be directly activated by dsRNA (and CpG-DNA), which can lead to enhanced killing of virus-infected cells by the NK cells [62-64]. Therefore, the response to the pathogen-associated molecular patterns produced by a virus is complex and is mediated by a range of different PRRs and cell types. There is likely to be a certain redundancy in the system; however, it is also clear that distinct PRRs mediate specific aspects of the antiviral response.

with IFN production [65], which is a common viral evasion strategy.

\section{Future perspectives}

The specific role of TLR3 in the complex picture of the antiviral response is still largely unclear. Mediating

\section{Box 1. Factors that influence the relevance of TLR3 for an antiviral response}

The relative contribution of TLR3 to the host response against a virus might depend on the following factors:

- Tropism of the virus (tissue cells or APCs?)

- Entry route of the virus (via endosomes or plasma membrane?)

- Localization of dsRNA (genome or replication intermediates) in the cell

- Nature of dsRNA in the cell: is it coated or packaged?

- Abundance of dsRNA intermediates produced during replication cycle

- Production of type I IFNs by virally infected tissue cells (does the virus have the means to interfere with IFN production?)

- Role of NK cells in the clearance of the virus crosspriming might turn out to be the "unique selling point' of TLR3, considering that TLR9 is not expressed on human myeloid DCs, and therefore probably has no role in crosspriming in humans. Some factors that can influence the relative relevance of TLR3 versus cytoplasmic dsRNA PRRs are summarized in Box 1 . The roles of the different DC populations in antiviral immunity (and in general) have not yet been clarified sufficiently, however, they are likely to hold important insights, considering the relatively clear-cut expression pattern of TLRs. Furthermore, a detailed investigation of the signaling pathways and the gene profiles induced by TLR3 and RIG-I will provide us with a better understanding of their respective roles. Open questions regarding the molecular aspects of the response to dsRNA are summarized in Box 2. It is also probable that we do not yet know the full picture of the response to dsRNA, and that additional players will be discovered. However, the fact that two distinct viruses, VV and HCV, have now been demonstrated to possess evasion strategies targeting the TLR3 pathway, strongly suggests 


\section{Box 2. Unresolved issues regarding the molecular response to dsRNA}

Is TRAF6 involved in TLR3-mediated signaling to NF- $\mathrm{KB}$ ?

Are there additional TRIF-independent components in the TLR3 pathway?

Are there additional cytoplasmic dsRNA receptors?

Do the pathways recognizing dsRNA display some crosstalk (e.g. involvement of PKR or mda-5 in TLR3 signaling) $[17,71]$ ?

What is the gene product of the identified mouse locus implied in the

TLR3- and PKR-independent response to dsRNA [41]?

How do the signals elicited by TLR3 and RIG-I differ?

that our appreciation of the role of TLR3 in the antiviral response can only grow.

\section{Acknowledgements}

Work in our laboratory is supported by Science Foundation Ireland, The Health Research Board and the Irish Research Council for Science, Education and Technology (IRCSET).

\section{References}

1 Alexopoulou, L. et al. (2001) Recognition of double-stranded RNA and activation of NF- $\kappa$ B by Toll-like receptor 3. Nature 413, 732-738

2 Edelmann, K.H. et al. (2004) Does Toll-like receptor 3 play a biological role in virus infections? Virology 322, 231-238

3 Hochrein, H. et al. (2004) Herpes simplex virus type-1 induces IFN- $\alpha$ production via Toll-like receptor 9-dependent and -independent pathways. Proc. Natl. Acad. Sci. U. S. A. 101, 11416-11421

4 Lund, J. et al. (2003) Toll-like receptor 9-mediated recognition of Herpes simplex virus-2 by plasmacytoid dendritic cells. J. Exp. Med. 198, 513-520

5 Lund, J.M. et al. (2004) Recognition of single-stranded RNA viruses by Toll-like receptor 7. Proc. Natl. Acad. Sci. U. S. A. 101, 5598-5603

6 Tabeta, K. et al. (2004) Toll-like receptors 9 and 3 as essential components of innate immune defense against mouse cytomegalovirus infection. Proc. Natl. Acad. Sci. U. S. A. 101, 3516-3521

7 Krug, A. et al. (2004) TLR9-dependent recognition of MCMV by IPC and DC generates coordinated cytokine responses that activate antiviral NK cell function. Immunity 21, 107-119

8 Krug, A. et al. (2004) Herpes simplex virus type 1 activates murine natural interferon-producing cells through Toll-like receptor 9. Blood 103, 1433-1437

9 Yoneyama, M. et al. (2004) The RNA helicase RIG-I has an essential function in double-stranded RNA-induced innate antiviral responses. Nat. Immunol. 5, 730-737

10 Takeda, K. and Akira, S. (2004) TLR signaling pathways. Semin. Immunol. 16, 3-9

11 Sato, S. et al. (2003) Toll/IL-1 receptor domain-containing adaptor inducing IFN- $\beta$ (TRIF) associates with TNF receptor-associated factor 6 and TANK-binding kinase 1, and activates two distinct transcription factors, NF- $\kappa \mathrm{B}$ and IFN-regulatory factor-3, in the Toll-like receptor signaling. J. Immunol. 171, 4304-4310

12 Moynagh, P.N. TLR signalling and activation of IRFs: revisiting old friends from the NF- $\mathrm{B}$ pathway. Trends Immunol. (in press) doi: 10.1016/j.it.2005.06.009

13 Jiang, Z. et al. (2004) Toll-like receptor 3-mediated activation of NF-кB and IRF3 diverges at Toll-IL-1 receptor domain-containing adapter inducing IFN-B. Proc. Natl. Acad. Sci. U. S. A. 101, 3533-3538

14 Hoebe, K. et al. (2003) Identification of Lps2 as a key transducer of MyD88-independent TIR signalling. Nature 424, 743-748

15 Yamamoto, M. et al. (2003) Role of adaptor TRIF in the MyD88independent Toll-like receptor signaling pathway. Science 301, 640-643

16 Oshiumi, H. et al. (2003) TICAM-1, an adaptor molecule that participates in Toll-like receptor 3-mediated interferon- $\beta$ induction. Nat. Immunol. 4, 161-167

17 Jiang, Z. et al. (2003) Poly(I-C)-induced Toll-like receptor 3 (TLR3)mediated activation of $\mathrm{NF} \kappa \mathrm{B}$ and MAP kinase is through an interleukin-1 receptor-associated kinase (IRAK)-independent pathway employing the signaling components TLR3-TRAF6-TAK1TAB2-PKR. J. Biol. Chem. 278, 16713-16719

18 Sarkar, S.N. et al. (2003) Double-stranded RNA signaling by Toll-like receptor 3 requires specific tyrosine residues in its cytoplasmic domain. J. Biol. Chem. 278, 4393-4396

19 Sen, G.C. and Sarkar, S.N. (2005) Transcriptional signaling by doublestranded RNA: role of TLR3. Cytokine Growth Factor Rev. 16, 1-14

20 Sarkar, S.N. et al. (2004) Novel roles of TLR3 tyrosine phosphorylation and PI3 kinase in double-stranded RNA signaling. Nat. Struct. Mol. Biol. 11, 1060-1067

21 Arbibe, L. et al. (2000) Toll-like receptor 2-mediated NF- $\mathrm{KB}$ activation requires a Rac1-dependent pathway. Nat. Immunol. 1, 533-540

22 Fitzgerald, K.A. et al. (2003) IKK $\varepsilon$ and TBK1 are essential components of the IRF3 signaling pathway. Nat. Immunol. 4, 491-496

23 Meylan, E. et al. (2004) RIP1 is an essential mediator of Toll-like receptor 3-induced NF-кB activation. Nat. Immunol. 5, 503-507

24 Han, K.J. et al. (2004) Mechanisms of the TRIF-induced interferonstimulated response element and NF- $\mathrm{BB}$ activation and apoptosis pathways. J. Biol. Chem. 279, 15652-15661

25 Gohda, J. et al. (2004) Cutting edge: TNFR-associated factor (TRAF) 6 is essential for MyD88-dependent pathway but not Toll/IL-1 receptor domain-containing adaptor-inducing IFN- $\beta$ (TRIF)-dependent pathway in TLR signaling. J. Immunol. 173, 2913-2917

26 Kaiser, W.J. and Offermann, M.K. (2005) Apoptosis induced by the Toll-like receptor adaptor TRIF is dependent on its receptor interacting protein homotypic interaction motif. J. Immunol. 174, $4942-4952$

27 Oshiumi, H. et al. (2003) TIR-containing adapter molecule (TICAM)-2, a bridging adapter recruiting to Toll-like receptor 4 TICAM-1 that induces interferon- $\beta$. J. Biol. Chem. 278, 49751-49762

28 Wietek, C. et al. (2003) Interferon regulatory factor-3-mediated activation of the interferon-sensitive response element by Toll-like receptor (TLR) 4 but not TLR3 requires the p65 subunit of NF- $\mathrm{KB}$. J. Biol. Chem. 278, 50923-50931

29 Doyle, S.E. et al. (2003) Toll-like receptor 3 mediates a more potent antiviral response than Toll-like receptor 4. J. Immunol. 170, 3565-3571

30 McWhirter, S.M. et al. (2004) IFN-regulatory factor 3-dependent gene expression is defective in Tbk1-deficient mouse embryonic fibroblasts. Proc. Natl. Acad. Sci. U. S. A. 101, 233-238

31 Servant, M.J. et al. (2003) Identification of the minimal phosphoacceptor site required for in vivo activation of interferon regulatory factor 3 in response to virus and double-stranded RNA. J. Biol. Chem. 278, 9441-9447

32 Rudd, B.D. et al. (2005) Differential role for TLR3 in respiratory syncytial virus-induced chemokine expression. J. Virol. 79, 3350-3357

33 Guillot, L. et al. (2005) Involvement of Toll-like receptor 3 in the immune response of lung epithelial cells to double-stranded RNA and influenza A virus. J. Biol. Chem. 280, 5571-5580

34 Wang, T. et al. (2004) Toll-like receptor 3 mediates West Nile virus entry into the brain causing lethal encephalitis. Nat. Med. 10, 1366-1373

35 Farina, C. et al. (2005) Preferential expression and function of Toll-like receptor 3 in human astrocytes. J. Neuroimmunol. 159, 12-19

36 Yang, E. et al. (2004) Cloning of TLR3 isoform. Yonsei Med. J. 45, 359-361

37 Diebold, S.S. et al. (2004) Innate antiviral responses by means of TLR7-mediated recognition of single-stranded RNA. Science 303, 1529-1531

38 Heil, F. et al. (2004) Species-specific recognition of single-stranded RNA via Toll-like receptor 7 and 8. Science 303, 1526-1529

39 Saunders, L.R. and Barber, G.N. (2003) The dsRNA binding protein family: critical roles, diverse cellular functions. FASEB $J$. 17, 961-983

40 Diebold, S.S. et al. (2003) Viral infection switches non-plasmacytoid dendritic cells into high interferon producers. Nature 424, 324-328

41 Hoebe, K. et al. (2003) Upregulation of costimulatory molecules induced by lipopolysaccharide and double-stranded RNA occurs by Trif-dependent and Trif-independent pathways. Nat. Immunol. 4, 1223-1229

42 Sumpter, R., Jr. et al. (2005) Regulating intracellular antiviral defense and permissiveness to hepatitis C virus RNA replication through a cellular RNA helicase, RIG-I. J. Virol. 79, 2689-2699 
43 Foy, E. et al. (2005) Control of antiviral defenses through hepatitis C virus disruption of retinoic acid-inducible gene-I signaling. Proc. Natl. Acad. Sci. U. S. A. 102, 2986-2991

44 Harte, M.T. et al. (2003) The poxvirus protein A52R targets Toll-like receptor signaling complexes to suppress host defense. J. Exp. Med. 197, 343-351

45 Stack, J. et al. (2005) Vaccinia virus protein A46R targets multiple Toll-like-interleukin-1 receptor adaptors and contributes to virulence. J. Exp. Med. 201, 1007-1018

46 Breiman, A. et al. (2005) Inhibition of RIG-I-dependent signaling to the interferon pathway during hepatitis $\mathrm{C}$ virus expression and restoration of signaling by IKK $\varepsilon$. J. Virol. 79, 3969-3978

47 Ferreon, J.C. et al. (2005) Molecular determinants of TRIF proteolysis mediated by the hepatitis $\mathrm{C}$ virus NS3/4A protease. J Biol Chem. 280, 20483-20492

$48 \mathrm{Li}, \mathrm{K}$. et al. (2005) Immune evasion by hepatitis C virus NS3/4A protease-mediated cleavage of the Toll-like receptor 3 adaptor protein TRIF. Proc. Natl. Acad. Sci. U. S. A. 102, 2992-2997

49 Matsumoto, M. et al. (2003) Subcellular localization of Toll-like receptor 3 in human dendritic cells. J. Immunol. 171, 3154-3162

50 Lopez, C.B. et al. (2004) TLR-independent induction of dendritic cell maturation and adaptive immunity by negative-strand RNA viruses. J. Immunol. 173, 6882-6889

51 Hemmi, H. et al. (2004) The roles of two I $\mathrm{B}$ kinase-related kinases in lipopolysaccharide and double stranded RNA signaling and viral infection. J. Exp. Med. 199, 1641-1650

52 Perry, A.K. et al. (2004) Differential requirement for TANK-binding kinase-1 in type I interferon responses to Toll-like receptor activation and viral infection. J. Exp. Med. 199, 1651-1658

53 Gil, J. and Esteban, M. (2000) Induction of apoptosis by the dsRNA-dependent protein kinase (PKR): mechanism of action. Apoptosis 5, 107-114

$54 \mathrm{Hsu}$, L.C. et al. (2004) The protein kinase PKR is required for macrophage apoptosis after activation of Toll-like receptor 4. Nature 428, 341-345

55 Iordanov, M.S. et al. (2005) Recruitment of TRADD, FADD, and caspase 8 to double-stranded RNA-triggered death inducing signaling complexes (dsRNA-DISCs). Apoptosis 10, 167-176

56 Matsumoto, S. et al. (2005) Analysis of dsRNA-induced apoptosis pathways using IFN response-noninducible siRNA-expression vector library. J. Biol. Chem. DOI: 10.1074/jbc.M412784200 (http://intl.jbc.org/)

57 Elco, C.P. et al. (2005) Analysis of genes induced by Sendai virus infection of mutant cell lines reveals essential roles of interferon regulatory factor $3, \mathrm{NF}-\kappa \mathrm{B}$, and interferon but not Toll-like receptor 3 . J. Virol. 79, 3920-3929

58 Degli-Esposti, M.A. and Smyth, M.J. (2005) Close encounters of different kinds: dendritic cells and NK cells take centre stage. Nat. Rev. Immunol. 5, 112-124

59 Reis e Sousa, C. (2004) Toll-like receptors and dendritic cells: for whom the bug tolls. Semin. Immunol. 16, 27-34

60 Ito, T. et al. (2002) Roles of Toll-like receptors in natural interferonproducing cells as sensors in immune surveillance. Hum. Immunol. $63,1120-1125$

61 Wagner, H. (2004) The immunobiology of the TLR9 subfamily. Trends Immunol. 25, 381-386

62 Pisegna, S. et al. (2004) p38 MAPK activation controls the TLR3mediated up-regulation of cytotoxicity and cytokine production in human NK cells. Blood 104, 4157-4164

63 Schmidt, K.N. et al. (2004) APC-independent activation of NK cells by the Toll-like receptor 3 agonist double-stranded RNA. J. Immunol. $172,138-143$

64 Sivori, S. et al. (2004) CpG and double-stranded RNA trigger human NK cells by Toll-like receptors: induction of cytokine release and cytotoxicity against tumors and dendritic cells. Proc. Natl. Acad. Sci. U. S. A. 101, 10116-10121

65 Schulz, O. et al. (2005) Toll-like receptor 3 promotes cross-priming to virus-infected cells. Nature 433, 887-892

66 Fujimoto, C. et al. (2004) Polyriboinosinic polyribocytidylic acid [poly(I:C)]/TLR3 signaling allows class I processing of exogenous protein and induction of HIV-specific $\mathrm{CD}^{+}$cytotoxic T lymphocytes. Int. Immunol. 16, 55-63

67 Datta, S.K. et al. (2003) A subset of Toll-like receptor ligands induces cross-presentation by bone marrow-derived dendritic cells. J. Immunol. 170, 4102-4110

68 Sato, A. and Iwasaki, A. (2004) Induction of antiviral immunity requires Toll-like receptor signaling in both stromal and dendritic cell compartments. Proc. Natl. Acad. Sci. U. S. A. 101, 16274-16279

69 Lebre, M.C. et al. (2003) Double-stranded RNA-exposed human keratinocytes promote Th1 responses by inducing a type- 1 polarized phenotype in dendritic cells: role of keratinocyte-derived tumor necrosis factor $\alpha$, type I interferons, and interleukin-18. J. Invest. Dermatol. 120, 990-997

70 Le Bon, A. et al. (2003) Cross-priming of $\mathrm{CD}^{+} \mathrm{T}$ cells stimulated by virus-induced type I interferon. Nat. Immunol. 4, 1009-1015

$71 \mathrm{Li}, \mathrm{K}$. et al. (2005) Distinct poly(I-C) and virus-activated signaling pathways leading to interferon- $\beta$ production in hepatocytes. $J$ Biol Chem. 280, 16739-16747

\section{Free journals for developing countries}

The WHO and six medical journal publishers have launched the Access to Research Initiative, which enables nearly 70 of the world's poorest countries to gain free access to biomedical literature through the Internet.

The science publishers, Blackwell, Elsevier, the Harcourt Worldwide STM group, Wolters Kluwer International Health and Science, Springer-Verlag and John Wiley, were approached by the WHO and the British Medical Journalin 2001. Initially, more than 1000 journals will be available for free or at significantly reduced prices to universities, medical schools, research and public institutions in developing countries. The second stage involves extending this initiative to institutions in other countries.

Gro Harlem Brundtland, director-general for the WHO, said that this initiative was 'perhaps the biggest step ever taken towards reducing the health information gap between rich and poor countries'.

See http://www.healthinternetwork.net for more information. 